\title{
Wandering Spleen in a Young Primiparous Female Patient Treated by Laparoscopic Splenectomy: A Case Report
}

Jad J Terro', Bilal El-Chamaa², Sary Abdallah ${ }^{3}$, Kassem Jammoul', Rayyan El Lakkis ${ }^{1}$, Etienne ElHelou $^{1}$, Mohammad Ahmad El-Raishouni ${ }^{4}$, Abbass Shibli', Jaafar Al-Shami ${ }^{1}$, Houssam Khodor Abtar

${ }^{1}$ Department of General Surgery, Faculty of Medical Sciences at Lebanese University, Beirut, Lebanon ${ }^{2}$ Department of General Surgery, Hotel-Dieu de France University Hospital, Saint-Joseph University, Beirut, Lebanon

${ }^{3}$ Department of General Surgery, Rayak Hospital, Rayak, Beirut, Lebanon

${ }^{4}$ FAHS Surgical Services, Michigan, USA

${ }^{5}$ Department of General Surgery, Central Military Hospital, Beirut, Lebanon

Corresponding Author: Jad J Terro, Beirut, Lebanon, j.terro@hotmail.com

Department of General Surgery, Rayak Hospital, Rayak, Lebanon

Financial support: None

Conflict of Interest: None

\begin{abstract}
Background: Wandering spleen (WS) is an uncommon splenic disorder defined as the displacement of the spleen from its usual anatomical position. It is most prevalent in females in their reproductive age. It can be encountered incidentally or may present with symptoms. Risks of complications exist and vary with the presentation. Several imaging techniques are able to define it clearly preoperatively. Laparoscopic surgery is the definitive diagnostic and therapeutic method.

Case Report: A 24-year-old pregnant female patient presented to the Emergency Department complaining of intermittent dull abdominal pain and pelvic heaviness for 9 days. ContrastEnhanced Computed Tomography and a color Doppler sonography of the abdomen and pelvis showed a large-sized spleen in the pelvis corresponding to a diagnosis of WS, without ischemic signs. The patient underwent a laparoscopic splenectomy and was vaccinated for Haemophilus influenza, meningococcus, and pneumococcus postoperatively. The post-operative period was uneventful, and the patient later delivered a full-term baby girl by a Cesarean section.

Conclusion: This is, to the best of our knowledge, the second reported case of laparoscopic splenectomy in a pregnant patient with WS. While splenopexy is associated with better outcomes, splenectomy seems to be preferred in pregnant patients, who may undergo a vaginal delivery, but guidelines and data are still scarce regarding the management of WS in pregnancy. Vaccination against encapsulated bacteria is required in case of splenectomy.

Keywords: Wandering spleen, splenectomy, splenopexy, pregnant patient, intermittent abdominal pain, palpable abdominal mass, case report.
\end{abstract}




\section{Background}

Wandering Spleen (WS) is an entity that was first described by a Dutch physician in 1667. It is a rare anatomical disorder in which the displacement and free movement of the spleen from its usual anatomical position into the lower abdomen or pelvis occurs. Its pathophysiologic mechanism is poorly understood, and it has been attributed to both acquired and congenital disorders. In the adult population, it most commonly affects multiparous female patients between 20 to 40 years old [1].

Clinical presentation of WS ranges from asymptomatic to acute abdomen. While it may be an incidental finding, it may also be lifethreatening, especially in pregnant women, with several potential complications such as splenic infarction, sepsis, pancreatitis, gastrointestinal varices, splenic vein thrombosis, and splenic rupture [2]. Color Doppler sonography and Contrast-Enhanced Computed Tomography scan (CECT scan) of the abdomen and pelvis have an indispensable role in diagnosing WS. Surgery is the treatment of choice, and depending on the case, a splenopexy or a splenectomy is performed [3].

In this article, we present the case of a primiparous 24-year-old female patient found to have a WS with partial torsion at her 6th-week of gestation. The patient underwent a laparoscopic splenectomy and was discharged home after an uneventful hospital stay. To the best of our knowledge, this is the second reported case of laparoscopic splenectomy in a pregnant female patient with WS in English literature.

\section{Case Presentation}

This is the case of a previously healthy 24-yearold female patient, with an obstetric resumé of G1P1A0 presenting to the emergency department at 6 weeks gestational age. She complained of an intermittent, diffuse, and dull abdominal pain, with increasing intensity, and pelvic heaviness for 9 days. Her pain was initially mild, and her obstetrician-gynecologist attributed it to pregnancy. She had no previous surgical or relevant family history but a history of anorexia and undocumented weight loss. The patient denied nausea, vomiting, constipation, and abdominal distension. She was hemodynamically stable.

On physical examination, she was afebrile, her body-mass index was 22.3, she looked ill and in pain, her abdomen was non-distended, soft, and there was a palpable tender mass in the suprapubic area extending to the left lower quadrant. She was regularly visiting her obstetrician and her pregnancy had been uncomplicated with no obstetric related complaints.

Blood tests revealed a low hemoglobin level (10 g/dL) (Normal Range [NR]: 12.5-14.5 g/dL), low platelets $\left(100 \times 10^{9} / \mathrm{L}\right)$ (NR: 150 to $\left.400 \times 10^{9} / \mathrm{L}\right)$ and a normal white blood cell count.

An abdominal-pelvic Computed Tomography scan (CT scan) with intravenous contrast showed a missing spleen in the left upper quadrant with displacement to the pelvic region (Fig.1A-B), heterogeneity on arterial phase, measuring $16 \mathrm{~cm}$ on its maximal diameter and with no thrombotic lesions on its vessels (Fig. 1C).

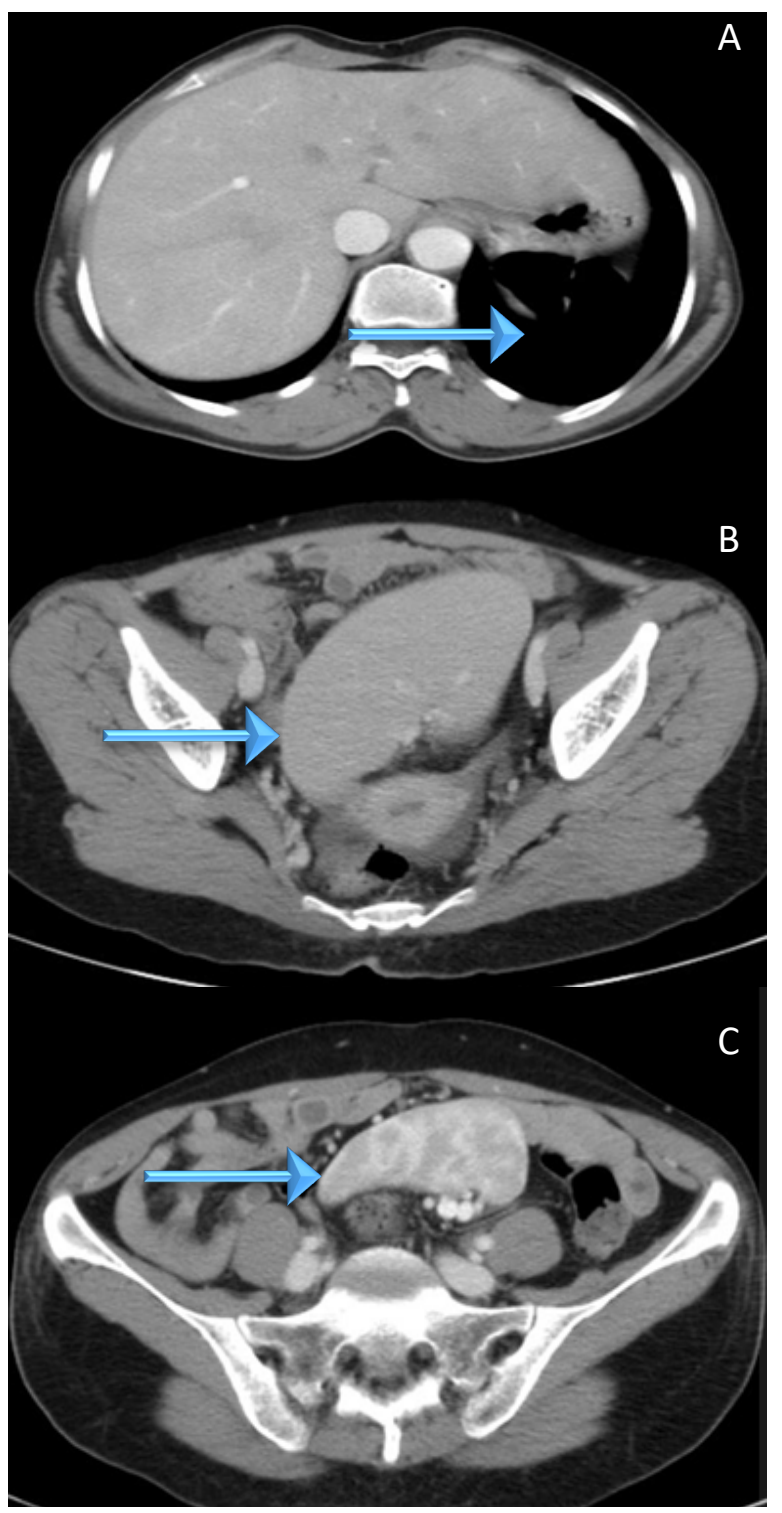

Figure 1: (A) CECT scan showing the absence of a spleen in the left hypochondrium, (B) the spleen in the middle pelvic region and (C) a heterogenous spleen with no vascular thrombosis 
The patient was admitted to the regular floor. On the next day, a Color Doppler Ultrasound showed a normal gestational sac, and the spleen was noted in the pelvic region with no torsion and normal blood flow.

After consulting her obstetrician, we decided to perform a laparoscopic exploration. We inserted 5,10 , and $12 \mathrm{~mm}$ trocars on the left paraumbilical, umbilical, and right paraumbilical region, respectively. Splenomegaly and spleniccongestion were seen with partial twisting at its hilum. We opted for a laparoscopic splenectomy (Fig. 2). The spleen was not adherent to any abdominal organ, we divided the hilum with an EndoGIA $60 \mathrm{~mm}$ white, and retrieved the spleen in an Endocatch bag after morcellation. The total operative time was 45 minutes.

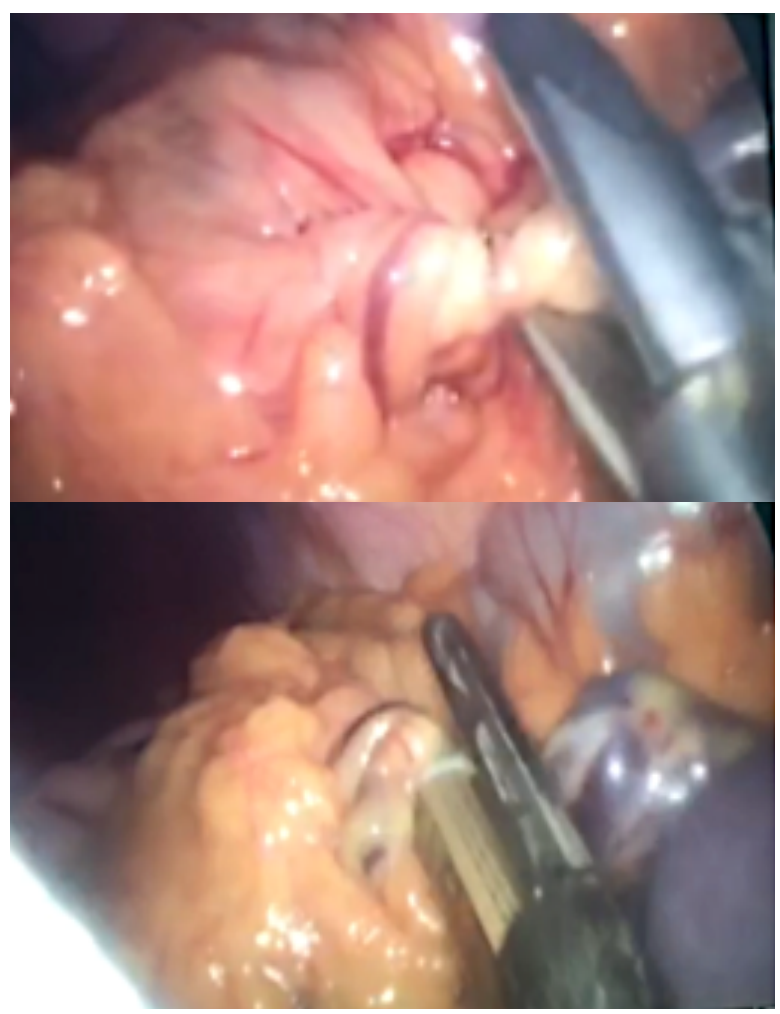

Figure 2: Laparoscopic evaluation showing a pelvic nonadherent spleen suspended by its partially torted pedicle

The patient had an uneventful postoperative hospital course. She took the Neisseria meningitides, Haemophilus influenza, and pneumococcal vaccinations, and was discharged home on postoperative day 5 , tolerating a regular diet. Her scheduled follow-ups with her obstetrician and the surgeon were smooth. Histopathology showed a congested spleen with microinfarcts at different sites and no signs of malignancies. She gave birth to a full-term healthy girl through an elective Cesarean section.

\section{Discussion}

WS is a rare entity with a debatable etiology. It can be congenital or acquired. The spleen is normally fixed in its position by three main ligaments: the gastrosplenic, splenorenal, and phrenicocolic ligaments in addition to other ligaments such as the pancreaticosplenic, pancreaticocolic, phrenicosplenic and splenocolic ligaments. Laxity or malformation of these ligaments is conventionally the leading cause of a WS. Congenital causes lead to pathologic dorsal mesogastrium development such as in connective tissue disease. Acquired causes include trauma, pregnancy (due to the effect of estrogens), Hodgkin lymphoma, malaria, multiparity, infectious mononucleosis, and left kidney enlargement or agenesis [3, 4].

The female to male ratio of WS depends on the age group, it is 1:2.5 in age $<1$ year, approximately $1: 1$ in the age range of 1 and 10 years, and $7: 1$ in those $>10$ years. The commonest age of presentation is $<1$ year of age, followed by females in their third decade of life. The incidence of WS is not well documented because many cases are asymptomatic, however, it is found in $0.2 \%$ of splenectomies [2].

The classic triad for clinical diagnosis of WS is defined by Gindrey and Piquard as a palpable abdominal mass, painless mobility if directed towards the left upper quadrant and painful if in opposite direction, and left hypochondrial tympany. When chronically symptomatic in adults, it presents mostly as an abdominal mass with or without abdominal pain and gastrointestinal complaints due to intermittent pedicle torsion-detorsion episodes [3].

Besides the mass effect of the displaced spleen on the bowels, the chronic pedicle torsion leads to pancreatic tail involvement, splenomegaly, congestion, and portal hypertension with gastroesophageal varices. Furthermore, splenomegaly leads to more traction on the splenic pedicle, more displacement of the spleen, and more risk for torsion [4]. The situation is harsher when the patient comes with an acute surgical abdomen and the most feared and frequent complication is complete pedicle torsion (60\%) [1]. This may lead to splenic necrosis and subcapsular hematoma, acute pancreatitis, sepsis, variceal bleeding, and splenic vein thrombosis [3]. Other complications are splenic rupture, spontaneously or due to trauma, intestinal obstruction due to mass effect, and 
gastric volvulus. The mortality rate reaches $50 \%$ in complicated WS [5].

Early diagnosis is crucial because of the possible complications. Preoperative parameters are needed in diagnosis and surgical planning. Laboratory tests aren't usually helpful but may show leukocytosis, thrombocytopenia, or HowellJolly bodies on peripheral smear [3, 6]. Enhanced CT scan can be the first imaging modality of choice, as it gives us the size of the spleen, site, adherences, involvement of other organs, pedicle length and density, and whether there is torsion or not. It may also detect the presence of a thrombus in the torted pedicle showing a pathognomonic "whirl-sign". Most commonly, the CT scan will show the absence of a spleen at the usual site and the presence of an ectopic comma-shaped spleen with possible pedicular torsion. Chronic and intermittent torsion can be also seen on the enhanced CT scan and is denoted by a peri-splenic pseudocapsule formed due to collateral circulations because of indolent ischemia [7].

Ultrasonography of the abdomen and pelvis paired with Colored Doppler is an excellent tool in determining the size and position of the spleen, together with any vascular compromise in the case of pedicle torsion. KUB is less important, it can show the presence of a mass with possible distended bowel loops in case of partial obstruction. When available, MRI is a good modality with equal diagnostic importance as the CT scan [5]. In our case, despite the great value of ultrasonography as an imaging modality in a pregnant patient, we opted for a CT scan of the abdomen and pelvis with the approval of the obstetrician, since there is no available MRI and no radiologist during the nightshift at this peripheral hospital.

Surgery is the golden standard for definitive diagnosis of WS, with a preference for laparoscopic surgery. Several presentations exist in different age groups, and they may affect the decision to do a splenopexy or a splenectomy. Conservative treatment is limited to some cases such as elderly patients, but the complication rate can reach $65 \%$. Knowing that the spleen has an indispensable role in immunity, lights are shed into splenopexy, especially in the pediatric population. Splenectomy was the recommended procedure previously, but due to increased rates of postoperative sepsis, splenopexy became preferable. Splenopexy is indicated in cases of WS with or without a torted pedicle in the absence of signs of vascular thrombosis or infarction, even in cases of acute abdomen. Many techniques are available in the literature such as simple suturing into the diaphragm or the abdominal wall, or the creation of a retroperitoneal pouch, with or without a mesh. In cases of splenic ischemia with infarction, rupture, or other associated visceral sufferings, splenectomy is a must. It is agreed also that the presence of an afunctional spleen, a large-sized WS, an endangered WS with long pedicle, or WS in a pregnant woman who may undergo a vaginal birth are also recommendations for splenectomy. Laparoscopy is a safe procedure at any time of pregnancy in the presence of an experienced surgeon.

To minimize the rates of post-splenectomy sepsis, vaccination by the triple vaccine is recommended 3 days before the procedure: Haemophilus influenzae, Meningococcus, and Pneumococcus $[3,5]$. The decision was taken to do a laparoscopic splenectomy in our patient for 1) the presence of a big congested WS, 2) pregnancy and labor risks on this pelvic WS, 3) hypersplenism, and 4) the shorter duration of splenectomy operation. In the case of our patient, the triple vaccine was given postoperatively due to its unavailability pre-operatively.

In our 24-year-old female patient who presented for intermittent abdominal pain with mild thrombocytopenia and anemia, diagnosed with a WS with splenomegaly and incomplete pedicle torsion, we decided to do a laparoscopic splenectomy.

Pathology results came with microinfarctions at different sites of the spleen, with no signs of malignancy.

\section{Conclusion}

This is the second case of a WS treated with laparoscopic splenectomy in a pregnant woman reported in English literature.

WS is not a daily encountered diagnosis. A high level of suspicion is required during the physical examination of such cases, and preoperative imaging diagnosis is essential, especially to assess vascular suffering before the planned procedure. Treatment is surgical. Splenopexy is superior to splenectomy, but data and guidelines are lacking in the case of a pregnant patient. Triple vaccination against encapsulated bacteria must be performed when opting for a splenectomy. 


\section{References}

1. Sojo-Rodríguez V, Cañete-Gómez J, Olivares C, Reguera-Rosal J, Segura-Sampedro JJ, CamachoMarente V, López-Bernal F, Padillo-Ruiz J. Acute abdomen due to torsion of the wandering spleen. Rev Esp Enferm Dig. 2015 Apr;107(4):229-30. PMID: 25824922.

2. Yücel E, Kurt Y, Ozdemir Y, Gun I, Yildiz M. Laparoscopic splenectomy for the treatment of wandering spleen in a pregnant woman: a case report. Surg Laparosc Endosc Percutan Tech. 2012 Apr;22(2):e102-4.

https://doi.org/10.1097/SLE.0b013e318246beb5.

PMID: 22487633.

3. Soleimani M, Mehrabi A, Kashfi A, Fonouni $H$, Büchler MW, Kraus TW. Surgical treatment of patients with wandering spleen: report of six cases with a review of the literature. Surg Today. 2007;37(3):261-9. https://doi.org/10.1007/s00595-006-3389-0. Epub 2007 Mar 9. PMID: 17342372.

4. Turhan A, Kapan S, Gonenc M, Dogan M, Aygun E. Wandering spleen: Report of two cases. Int Med Case Rep J. $2010 \quad$ Mar 9;3:19-22. https://doi.org/10.2147/imcrj.s8937. PMID: 23754883; PMCID: PMC3658214.

5. Iqbal M, Tareen MA, Sohail U. Torsion of a wandering spleen presenting as a case of acute abdomen. J Coll Physicians Surg Pak. 2009 Feb;19(2):123-4. PMID: 19208319.

6. Gilman RS, Thomas RL. Wandering spleen presenting as acute pancreatitis in pregnancy. Obstet Gynecol. $2003 \quad$ May;101(5 Pt 2):1100-2. https://doi.org/10.1016/s0029-7844(02)02617-0.

PMID: 12738115.

7. Priyadarshi RN, Anand U, Kumar B, Prakash V. Torsion in wandering spleen: CT demonstration of whirl sign. Abdom Imaging. 2013 Aug;38(4):835-8. https://doi.org/10.1007/s00261-012-9944-9. PMID: 22829098. 